\title{
Adequacy of Transthoracic Needle Biopsy Samples in the Diagnosis of a Peripheral Lung Lesion - Comparing Success Rates of Various Imaging Modalities
}

\author{
Salem Alshimemeri ${ }^{1}$, Hanaa Bamefleh ${ }^{2}$, Shukri Loutfi ${ }^{3}$, Yazeed Bindous ${ }^{3}$, Azzam Khankan ${ }^{3}$, \\ Waad Almusailhi ${ }^{4}$ \\ ${ }^{1}$ College of Medicine, King Saud bin Abdulaziz University for Health Sciences, Riyadh, Kingdom of Saudi Arabia \\ ${ }^{2}$ Department of Pathology and Laboratory Medicine, King Abdulaziz Medical City, Riyadh, Kingdom of Saudi Arabia \\ ${ }^{3}$ Department of Medical Imaging, King Abdulaziz Medical City, Riyadh, Kingdom of Saudi Arabia \\ ${ }^{4}$ College of Medicine, Almaarefa Colleges for Science and Technology, Riyadh, Kingdom of Saudi Arabia
}

\section{Email address:}

Salem.Alshimemeri@gmail.com (S. Alshimemeri), BameflehH@NGHA.MED.SA (H. Bamefleh), LoutfiS@NGHA.MED.SA (S. Loutfi), BinDousYa@NGHA.MED.SA (Y. Bindous), KhankanA@NGHA.MED.SA (A. Khankan), Waad-almusailhi@hotmail.com (W. Almusailhi)

\section{To cite this article:}

Salem Alshimemeri, Hanaa Bamefleh, Shukri Loutfi, Yazeed Bindous, Azzam Khankan, Waad Almusailhi. Adequacy of Transthoracic Needle Biopsy Samples in the Diagnosis of a Peripheral Lung Lesion - Comparing Success Rates of Various Imaging Modalities. International Journal of Medical Imaging. Vol. 5, No. 3, 2017, pp. 34-37. doi: 10.11648/j.jimi.20170503.11

Received: March 6, 2017; Accepted: March 30, 2017; Published: April 27, 2017

\begin{abstract}
The aim of the study was to test whether a difference in the imaging modality (CT, Fluoroscopy, or Ultrasound) would result in a higher biopsy success rate for the diagnosis of lung cancer. A total of 144 transthoracic needle biopsies performed under guidance of different imaging modalities were retrospectively reviewed at King Abdulaziz Medical city in Riyadh between 2008 and 2012. A biopsy was counted a success whenever a definitive diagnosis could be achieved. CT guided biopsies revealed 51 successes out of 86 total samples, fluoroscopy guided biopsies revealed 8 successes out of 20 total samples, while for ultrasound guided biopsies, 30 successes out of 38 biopsies gave a definitive diagnosis. Comparing CT guided biopsies vs. Fluoroscopy guided biopsies, we got a p-value of 0.1884 which is clinically insignificant, $95 \%$ Confidence Interval [-0.07628, 0.46233]. On comparison of CT guided biopsies vs. ultrasound guided biopsies the p-value was 0.05558 which is also clinically insignificant, 95\% Confidence Interval [-0.38150, -0.011399]. When ultrasound guided biopsies were compared to the fluoroscopy guided biopsies a p-value of $0.007461<0.025$ was achieved which is clinically highly significant, $95 \%$ Confidence Interval [-0.38150, -0.011399]. It was determined with $95 \%$ confidence that there is a clinically significant difference ( $p$-value of 0.007461 ) between success rates of Fluoroscopy guided biopsies and ultrasound guided biopsies, but not between the other pairs of modalities. Further investigations with larger sample size are warranted to compare the efficacy of fluoroscopy and ultrasound based imaging modalities for transthoracic needle biopsy.
\end{abstract}

Keywords: Small-Cell Lung Cancer, Non-small Cell Lung Cancer, Computed Tomography, CT-Fluoroscopy, Ultrasound, Transthoracic Needle Aspiration, PACS

\section{Introduction}

Lung cancer is the leading cause of cancer related deaths in the industrialized world [1]. According to the American Cancer Society, approximately 224,390 new cases of lung cancer will be diagnosed in the United Sates in the year 2016 and 158,080 deaths will be due to lung cancer related causes, accounting for $27 \%$ of all cancer deaths [2].
Lung cancer is classified into two groups; Small-cell lung cancer (SCLC) and non-small cell lung cancer (NSCLC). Squamous cell carcinoma, large-cell carcinoma, and adenocarcinoma are all classified under NSCLC [1]. Nearly 4.5-14 million CT scan examinations of the chest are done annually in the United States for the possible detection of lung cancer underlining the importance of definitive and safer diagnostic procedures. [3, 4] Pulmonary nodules are present not only in lung cancers but may also represent 
metastases of breast and colon cancers. Early detection of malignancy is important for treatment and with improvement in technology, identification of small lung nodules is now possible. [5-7]

Peripheral pulmonary nodules of suspicious nature present a dilemma. Choice of diagnostic procedure for biopsy of possible malignant lung nodules is made in accordance with several aspects; such as the size and location of tumour, accessibility of the lesion and the primary tumour, availability of the clinical expert and potential complications. A procedure that is well tolerated, can give maximum information and is minimally invasive to the patient is the most preferred technique. [6-10]

The diagnosis of lung cancer for patients with a peripheral lesion is established through transthoracic needle aspiration (TTNA) as recommended by the American College of Chest Physicians evidence-based clinical practice guidelines [11]. The TTNA is a biopsy technique that utilizes guidance by $\mathrm{CT}$, ultrasound and/or fluoroscopy to reach percutaneous peripheral nodules without relying on central airways. The biopsy can be taken with imaging guidance of fluoroscopy, $\mathrm{CT}$, or ultrasound depending on the site and position of the lesion, availability of the equipment, and preference of the operator [12]. A report by Manhire et al suggested that within TTNA, no association exists between complication rates and needle type and size [13]. TTNA has diagnostic yields of greater than $90 \%$, which is higher than any other non-surgical approach [14] Other viable diagnostic options for indeterminate pulmonary lesions such as flexible bronchoscopy and its ancillary procedures have lower success rates ranging from (14-63\%). [15]

The disadvantages of TTNA however, are very high rates of pneumothorax (ranging between15-25\%) while other bronchoscopic approaches have much lower rates $(\sim 1.5 \%)$. [16] Air embolism and tumour seeding are other complications associated with the technique. [16-18] Due to this reason, the American College of Chest Physicians recommends TTNA for lung nodules present without a bronchus sign only when RP-EBUS (radial endobronchial ultrasound) is not available. [13] Although transthoracic needle aspiration is a technically demanding procedure, documented evidence has shown that it is accurate with limited morbidity. [6,7, 10]

Image guidance is a crucial aspect of the TTNA technique. Currently computed tomography (CT), computed tomography fluoroscopy (CT-fluoroscopy) and ultrasound (US) guided procedures are most commonly used for imaging the biopsy area. Compared with conventional CT, Fluoroscopy guided transthoracic biopsies are considered superior because they require fewer needle passes. Moreover, use of Fluoroscopy considerably shortens the procedure time by up to $\sim 27 \%$. [14] The shorter procedure time and fewer needle passes also results in fewer complications being associated with Fluoroscopy guided biopsies. [14, 15] Various groups have shown lower pneumothorax rates for Fluoroscopy guided transthoracic needle biopsies. [14-18] The only drawback of using Fluoroscopy is the risk of exposure to radiation for both patients and radiologists, which can be minimized by using the 'quick check' method. $[20,21]$ Ultrasound guided TTNA biopsies are generally well tolerated probably because lung lesions guided by ultrasounds are peripheral in nature with less chances of pneumothorax. [22]

\section{Methods and Patients}

In this study, data from a total of 144 transthoracic needle biopsies carried out at King Abdulaziz Medical city in Riyadh, Saudi Arabia, during the period of four years, from 2008 to 2012, was retrospectively reviewed. The transthoracic needle biopsies were performed under the guidance of three different imaging modalities of CT, CTfluoroscopy and ultrasound. Data was extracted from the PACS (picture archiving and communication system) of the radiology department, all consecutive biopsies done during the period were included in the study, Core biopsies from the mediastinum or pleura were not included. Sampling was counted as being a 'success' if a definitive diagnosis of malignancy or a benign sample was obtained following cytology or microbiology report, whereas 'non-specific diagnosis' or 'inadequate size of sample' were counted as failures.

Choice of imaging module was made according to the size and location of lesion. Statistical analysis of differences in various imaging modalities was done using the $\chi 2$ test and reported as $P$ values. A $P$ value of $<.05$ was considered statistically significant.

\section{Results and Discussion}

\subsection{Patient Demographics}

Table 1 gives a summary of baseline characteristics and the demographic profile of the patients enrolled in the retrospective study. Of the total 144 patients, 97 were female and 47 were females. Mean age of patients was 66.20 years. Figure 1 ( $a, b$ and $c)$ describes the adequacy of TTNA biopsies for different patient profiles. The success rate were in general independent of gender and age.

Table 1. Demographic characteristics of the patients. Values are represented as frequency (\%). * $p$ values were generated comparing 1 category against the other category grouped as one.

\begin{tabular}{|c|c|c|c|c|c|c|c|}
\hline \multirow[b]{3}{*}{ Age mean years (SD) } & \multirow{2}{*}{\multicolumn{2}{|c|}{ All Samples n= 144}} & \multicolumn{5}{|c|}{ Adequacy of tissue samples obtained } \\
\hline & & & \multicolumn{2}{|c|}{ Success n=89 } & \multicolumn{2}{|c|}{ Failure $n=55$} & \multirow{2}{*}{$\begin{array}{l}\text { p value } \\
0.375\end{array}$} \\
\hline & 66.20 & $(11.51)$ & 66.88 & $(11.47)$ & 65.077 & $(11.61)$ & \\
\hline Gender & & & & & & & \\
\hline Male & 97 & $(67.40)$ & 60 & $(61.86)$ & 37 & $(38.14)$ & 0.986 \\
\hline
\end{tabular}




\begin{tabular}{|c|c|c|c|c|c|c|c|}
\hline \multirow[b]{3}{*}{ Female } & \multirow{2}{*}{\multicolumn{2}{|c|}{ All Samples n= 144}} & \multicolumn{5}{|c|}{ Adequacy of tissue samples obtained } \\
\hline & & & \multicolumn{2}{|c|}{ Success $n=89$} & \multicolumn{2}{|c|}{ Failure $n=55$} & \multirow[t]{2}{*}{ p value } \\
\hline & 47 & $(32.60)$ & 29 & $(61.70)$ & 18 & $(38.30)$ & \\
\hline Total & 144 & $(100.00)$ & & & & & \\
\hline \multicolumn{8}{|l|}{ Modality } \\
\hline $\mathrm{CT}$ & 86 & $(59.70)$ & 51 & $(59.30)$ & 35 & $(40.70)$ & $0.282 *$ \\
\hline Fluoro & 20 & $(13.90)$ & 8 & $(40.00)$ & 12 & $(60.00)$ & $0.029 *$ \\
\hline US & 38 & $(26.40)$ & 30 & $(78.95)$ & 8 & $(21.05)$ & $0.008 *$ \\
\hline Total & 144 & $(100.00)$ & & & & & \\
\hline
\end{tabular}

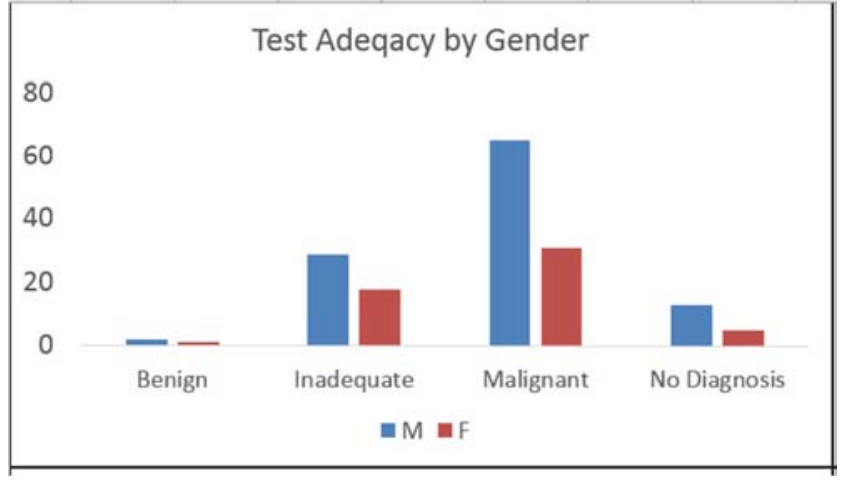

Figure 1a. Adequacy of TTNA biopsy procedure by gender.

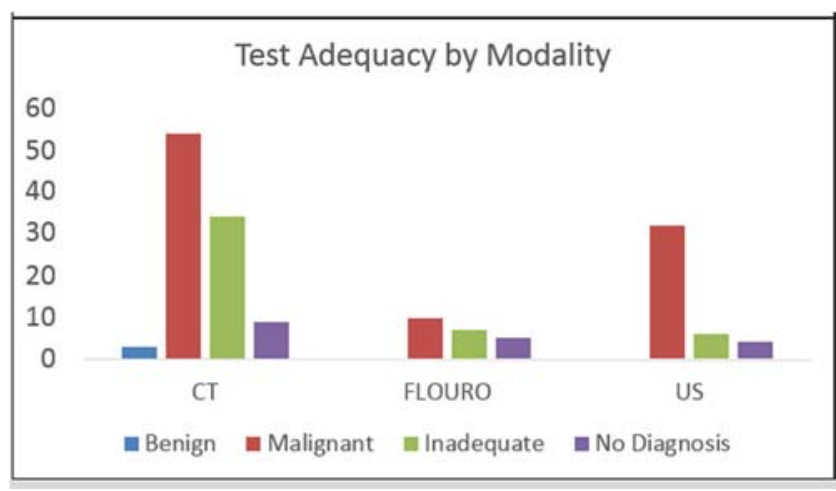

Figure $1 b$. Success rate of TTNA by the imaging modality used.

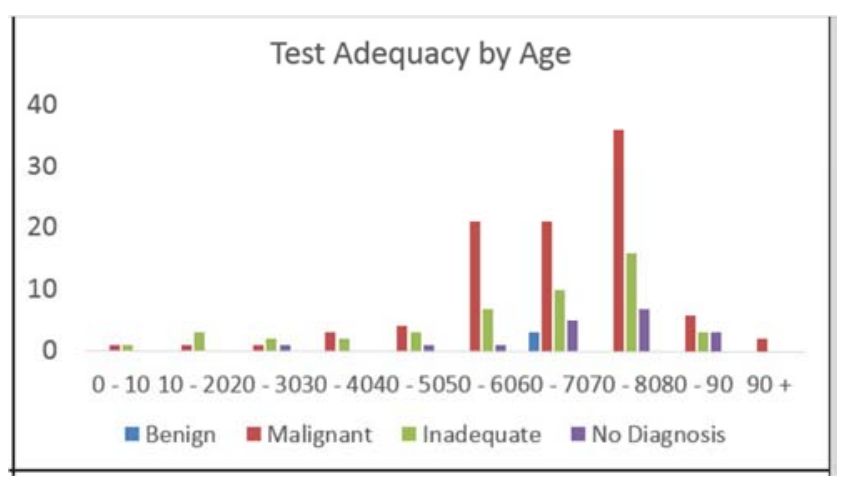

Figure 1c. Adequacy of the procedure as plotted against age of the patients. Data is plotted as frequency (\%).

\subsection{Success Rate of Different Imaging Modalities}

The success rate for conventional CT-guided biopsies was $\sim 59.3 \%$ (51 of 86 total samples), fluoroscopy guided biopsies revealed 8 successes out of 20 total samples (40\%), and ultrasound guided biopsies revealed a success rate of $78.9 \%$ (30 successes out of 38 total samples). Overall the diagnostic yield of image guided TTNA biopsy in our study was around $60 \%$ which is somewhat lesser than that in reported literature (70-90\%). [22] This could be due to the small sample size and/or very stringent conditions of counting a biopsy a success. Moreover, the diagnostic yield is also influenced by the size of the nodule with lobes $>2 \mathrm{~cm}$ giving better definitive diagnosis. [15] Other studies have also reported that for benign lesions, percutaneous lung biopsy shows low sensitivity and diagnostic accuracy (11-88\%). []

\subsection{Statistical Comparison of the Different Imaging Modalities}

Comparing CT guided biopsies (51 successes out of 86) vs. Fluoroscopy guided biopsies ( 8 successes out of 20) the p-value of 0.1884 was obtained which clinically insignificant, 95\% Confidence Interval [-0.07628, 0.46233].

When we compared CT guided biopsies (51 successes out of 86) vs. ultrasound guided biopsies (30 successes out of 38) the p-value was 0.05558 , which is also clinically insignificant, 95\% Confidence Interval [-0.38150, 0.011399].

On comparison of ultrasound guided biopsies (30 successes out of 38) vs. Fluoroscopy guided biopsies (8 successes out of 20) the p-value was $0.007461<0.025$ which is clinically highly significant, 95\% Confidence Interval [$0.38150,-0.011399$ ].

This study has all limitations inherent to retrospective, non-randomized studies. Therefore, we included only definitive diagnosis as established by the biopsy only and not any subsequent tests that may have been performed. We propose these interesting results are further investigated. A prospective study with a larger sample size designed specifically to study difference in fluoroscopy and ultrasound guided TTNA should be undertaken.

\section{Conclusion}

No significant differences were found between CT guided biopsies and Fluoroscopy guided biopsies or CT guided biopsies and ultrasound guided biopsies. However, the difference in the success rates of Fluoroscopy guided biopsies and ultrasound guided biopsies was a statistically significant one, which needs further testing with a larger sample size. It was determined with $95 \%$ confidence that there is a clinically significant difference ( $p$-value of 0.007461 ) between the Fluoroscopy guided biopsies and ultrasound guided biopsies modalities, but not between the other pairs of modalities. 


\section{Abbreviations Used}

(SCLC) Small-cell lung cancer

(NSCLC) Non-small cell lung cancer

(CT) Computed tomography

(CT-fluoroscopy) Computed tomography fluoroscopy

(US) Ultrasound

(TTNA) Transthoracic needle aspiration

PACS (picture archiving and communication system)

\section{References}

[1] Kumar V, Abbas AK, Fausto N, Mitchell RN. Robbins Basic Pathology. 8th ed. Philadelphia, PA: Saunders Elsevier; 2007.

[2] Siegel RL, Miller KD, Jemal A. Cancer statistics, 2016. CA Cancer J Clin. 2016 Jan;66(1):7-30. doi: 10.3322/caac.21332. Epub 2016 Jan 7.

[3] Stern SH. Nationwide Evaluation of X-ray Trends (NEXT): Tabulation and Graphical Summary of 2000 Survey of Computed Tomograph. Frankfort, KY: Conference of Radiation Control Program Directors, Inc; 2007.

[4] Aberle DR, Adams AM, Berg CD, et al; National Lung Screening Trial Research Team. Reduced lung-cancer mortality with low-dose computed tomographic screening. $N$ Engl J Med. 2011; 365 (5): 395-409.

[5] Winokur RS, Pua BB, Sullivan BW, Madoff DC. Percutaneous lung biopsy: technique, efficacy, and complications. Semin Intervent Radiol. 2013 Jun;30(2):121-7.

[6] Meyer CA," Transthoracic needle aspiration biopsy of benign and malignant lung lesions"--a commentary. AJR Am J of Roentgen. 2007 Apr;188(4):891-3.

[7] Gilbert C, Akulian J, Ortiz R, Lee H, Yarmus L. Novel bronchoscopic strategies for the diagnosis of peripheral lung lesions: Present techniques and future directions. Respirology. 2014 Jul;19(5):636-44. doi: 10.1111/resp.12301.

[8] DiBardino DM, Yarmus LB, Semaan RW. Transthoracic needle biopsy of the lung J Thorac Dis. 2015 Dec;7(Suppl 4): S304-16. doi: 10.3978/j.issn.2072-1439.2015.12.16.

[9] Lillington GA, Gould MK. Identification of benign pulmonary nodules by needle biopsy. Chest. 1998; 113:3-5.

[10] Rivera MP, Mehta AC, Wahidi MM. Establishing the diagnosis of lung cancer: Diagnosis and management of lung cancer, 3rd ed: American College of Chest Physicians evidence-based clinical practice guidelines. Chest. 2013 May;143(5 Suppl): e142S-65S. doi: 10.1378/chest.12-2353.

[11] Manhire A1, Charig M, Clelland C, Gleeson F, Miller R, Moss H, Pointon K, Richardson C, Sawicka E; BTS. Guidelines for radiologically guided lung biopsy. Thorax. 2003 Nov; 58(11):920-36.

[12] Detterbeck FC, Lewis SZ, Diekemper R, et al. Executive summary: diagnosis and management of lung cancer, 3rd:
American college of chest physicians evidence-based clinical practice guidelines. Chest 2013; 143:7S-37S.

[13] Gould MK, Fletcher J, Iannettoni MD, et al; American College of Chest Physicians. Evaluation of patients with pulmonary nodules: when is it lung cancer? ACCP evidencebased clinical practice guidelines (2nd edition). Chest. 2007; 132 ( suppl 3 ):108S-130S.

[14] Kim GR, Hur J, Lee SM, et al. CT fluoroscopy-guided lung biopsy versus conventional CT-guided lung biopsy: a prospective controlled study to assess radiation doses and diagnostic performance. Eur Radiol 2011;21(2):232-239.

[15] Heyer CM, Reichelt S, Peters SA, Walther JW, Müller KM, Nicolas V. Computed tomography-navigated transthoracic core biopsy of pulmonary lesions: which factors affect diagnostic yield and complication rates? Acad Radiol. 2008; 15 (8): 1017-1026.

[16] Wiener RS, Schwarts LM, Woloshin S, et al. Population-based risk for complications after transthoracic needle lung biopsy of a pulmonary nodule: an analysis of discharge records. Ann Intern Med 2011; 155:137-44.

[17] Tomiyama N, Yasuhara Y, Nakajima Y, et al. CT-guided needle biopsy of lung lesions: a survey of severe complication based on 9783 biopsies in Japan. Eur J Radiol 2006; 59:60-4.

[18] Fielding DI, Chia C, Nguyen P, et al. Prospective randomised trial of endobronchial ultrasound-guide sheath versus computed tomography guided percutaneous core biopsies for peripheral lung lesions. Intern Med J 2012; 42:894-900.

[19] Prosch H, Stadler A, Schilling M, et al. CT fluoroscopyguided vs. multislice CT biopsy mode-guided lung biopsies: accuracy, complications and radiation dose. Eur J Radiol 2012;81(5): 1029-1033.

[20] Paulson EK, Sheafor DH, Enterline DS, McAdams HP, Yoshizumi TT. CT fluoroscopy-guided interventional procedures: techniques and radiation dose to radiologists. Radiology 2001;220(1): 161-167.

[21] Heck SL, Blom P, Berstad A. Accuracy and complications in computed tomography fluoroscopy-guided needle biopsies of lung masses. Eur Radiol 2006;16(6):1387-1392.

[22] Sconfienza LM, Mauri G, Grossi F, et al. Pleural and peripheral lung lesions: comparison of US- and CT-guided biopsy. Radiology 2013; 266:930-5.

[23] Kothary, N, lock L, Sze, DL, Hofman LV. Computed tomography-guided percutaneous needle biopsy of pulmonary nodules: impact of nodule size on diagnostic accuracy. 2009 Sep;10(5):360-3. doi: 10.3816/CLC.2009. n.049.

[24] Lillington GA, Gould MK. Identification of benign pulmonary nodules by needle biopsy. Chest. 1998; 113:3-5.

[25] Khouri NF, Stitik FP, Erozan YS, et al. Transthoracic needle aspiration biopsy of benign and malignant lung lesions. AJR Am J Roentgenol. 1985; 144:281-288. 